\title{
High-resolution 21-cm observations of low-column density gas clumps in the Milky Way halo
}

\author{
N. Ben Bekhti ${ }^{1}$, P. Richter ${ }^{2}$, B. Winkel ${ }^{1}$, F. Kenn ${ }^{1}$, and T. Westmeier ${ }^{3}$ \\ 1 Argelander-Institut für Astronomie, Universität Bonn, Auf dem Hügel 71, 53121 Bonn, Germany \\ e-mail: [nbekhti; fkenn; bwinkel]@astro.uni-bonn.de \\ 2 Institut für Physik und Astronomie, Haus 28, Karl-Liebknecht-Str. 24/25, 14476 Golm (Potsdam), Germany \\ e-mail: prichter@astro.physik.uni-potsdam.de \\ 3 Australia Telescope National Facility, PO Box 76, Epping NSW 1710, Australia \\ e-mail: tobias.westmeier@csiro.au
}

Received 30 October 2008 / Accepted 12 June 2009

\begin{abstract}
Aims. We study the properties of low-column density gas clumps in the halo of the Milky Way based on high-resolution 21-cm observations.

Methods. Using interferometric data from the Westerbork Synthesis Radio Telescope (WSRT) and the Very Large Array (VLA), we studied H I emission at low-, intermediate- and high radial velocities along four lines of sight towards the quasars QSO J0003-2323, QSO B1331+170, QSO B0450-1310, and J081331+254503. Along these sightlines we had previously detected weak Ca II and Na I absorbers in the optical spectra of these quasars.

Results. The analysis of the high-resolution HI data reveals several compact and cold clumps of neutral gas at velocities similar to the optical absorption. The clumps have narrow H I line widths in the range of $1.8 \leq v_{\mathrm{FWHM}} \leq 13.0 \mathrm{~km} \mathrm{~s}^{-1}$, yielding upper limits for the kinetic temperature of the gas of $70 \leq T_{\max } \leq 3700 \mathrm{~K}$. The neutral gas has low HI column densities in the range of $5 \times 10^{18} \ldots 3 \times 10^{19} \mathrm{~cm}^{-2}$. All clumps have angular sizes of only a few arcminutes.

Conclusions. Our high-resolution 21-cm observations indicate that many of the Ca II and Na I absorbers seen in our optical quasar spectra are associated with low-column density Hi clumps on small angular scales. This suggests that, next to the massive, highcolumn density neutral gas clouds in the halo (the common $21-\mathrm{cm}$ low-, intermediate-, and high-velocity clouds, LVCs, IVCs, and HVCs), a population of low-mass, neutral gas structures exists in the halo and remain mostly unseen in the existing 21-cm all-sky surveys of IVCs and HVCs. One of our absorbers may be associated with the Magellanic Stream, two intermediate-velocity clouds are probably part of the Intermediate-Velocity Spur and the Low-latitude IV arch, respectively. The remaining systems could be located either in the lower halo or in the disk of the Milky Way. The estimated thermal gas pressures of the detected H I clumps are consistent with what is expected from theoretical models of gas in the inner and outer Milky Way halos.
\end{abstract}

Key words. Galaxy: halo - ISM: structure - quasars: absorption lines - galaxies: halos

\section{Introduction}

The disk of the Milky Way is surrounded by a gaseous halo that has an unknown extent. High-resolution absorption and emission line measurements have demonstrated that this halo contains a mixture of cool, warm, and hot gas (e.g., Savage \& Massa 1987; Majewski 2004; Fraternali et al. 2007, and references therein). Multi-wavelength observations of this gaseous material provide insight into the different processes of the exchange of gaseous matter and energy between the galaxy and the intergalactic medium. There is a permanent mass circulation between the Milky Way disk and the halo via the Galactic fountain process (Shapiro \& Field 1976; Bregman 1980; Shapiro \& Benjamin 1991) where supernova explosions expel material out of the disk into the halo, where it cools and then falls back onto the Galactic plane. This gas enriches the circumgalactic environment with heavy elements. Also, interactions with satellite galaxies inject interstellar material into the halo of the Milky Way (e.g., Mathewson et al. 1974). Finally, low-metallicity gas is most likely being accreted from the intergalactic medium (IGM, e.g., Wakker et al. 1999; Richter et al. 2001). These processes suggest that the gaseous material in the Milky Way halo is tightly connected to the ongoing formation and evolution processes of our galaxy.

A large fraction of the neutral gas in the halo is in the form of clouds with radial velocities inconsistent with a simple model of Galactic rotation (e.g., Lockman 2002; Lockman et al. 2002), the so-called intermediate- and high-velocity clouds (IVCs, HVCs, Muller et al. 1963). IVCs are most likely located in the lower Galactic halo at $d<2 \mathrm{kpc}$, whereas HVCs are thought to be located in the outer halo of galaxies at $5<d<50 \mathrm{kpc}$ (e.g., Sembach et al. 1991; van Woerden et al. 1999; Wakker 2001; Thom et al. 2006; Wakker et al. 2007, 2008).

Many IVCs and HVCs most likely have different origins, as expected from the various gas circulation processes in the halo outlined above. The bulk of IVCs are located close to the disk and appear to fall towards the Galactic plane, which could be explained by the galactic fountain model (Shapiro \& Field 1976; Bregman 1980; Shapiro \& Benjamin 1991). Some of the HVCs most likely emerge during interaction processes between the Milky Way and satellite galaxies due to merging and accretion. One prominent example is the Magellanic Stream, which appears to be a tidal feature from the Magellanic Clouds as they interact with the Milky Way (e.g., Mathewson et al. 1974). HVCs 
Table 1. Observational parameters of the high-resolution measurements with the WSRT and the VLA.

\begin{tabular}{cccccccccc}
\hline \hline Instrument & $\begin{array}{c}\text { Date } \\
{[\mathrm{m}: \mathrm{y}]}\end{array}$ & Configuration & $\begin{array}{c}l \\
{[\mathrm{deg}]}\end{array}$ & $\begin{array}{c}b \\
{[\mathrm{deg}]}\end{array}$ & $\begin{array}{c}\Omega_{\mathrm{HPBW}} \\
{[\mathrm{deg}]}\end{array}$ & $\begin{array}{c}\Theta_{\mathrm{HPBW}} \\
{\left[{ }^{\prime \prime}\right]}\end{array}$ & $\begin{array}{c}\delta v \\
{\left[\mathrm{~km} \mathrm{~s}^{-1}\right]}\end{array}$ & $\begin{array}{c}\Delta v \\
{[\mathrm{MHz}]}\end{array}$ & $\begin{array}{c}\sigma_{\text {rms }} \\
{\left[\mathrm{mJy} \mathrm{Beam}^{-1}\right]}\end{array}$ \\
\hline \multirow{2}{*}{ WSRT } & Feb. 2007 & $2 \times 48$ & 348.5 & 75.8 & 0.5 & $80 \times 180$ & 0.5 & 2.5 & 9.9 \\
& Feb. 2007 & $2 \times 48$ & 196.9 & 28.6 & 0.5 & $80 \times 120$ & 0.5 & 2.5 & 8.5 \\
\hline \multirow{2}{*}{ VLA } & Feb. 2007 & DnC & 49.4 & -78.6 & 0.5 & $100 \times 100$ & 1.3 & 1.56 & 10.6 \\
& Feb. 2007 & DnC & 211.8 & -32.1 & 0.5 & $100 \times 100$ & 1.3 & 1.56 & 13.3 \\
\hline
\end{tabular}

also have been brought into relation with primordial building blocks of galaxies in a hierarchical formation scenario (e.g., Oort 1966; Blitz et al. 1999).

A powerful method of analysing the characteristics of IVC and HVC gas at low redshifts is to analyse high-resolution ultraviolet (UV) and optical absorption spectra together with H I 21-cm emission data (Richter 2006; Ben Bekhti et al. 2008). The combination of these data provides important information about the spatial distribution and the physical properties of the clouds.

This paper is based on our previous work (Ben Bekhti et al. 2008), in which we analysed VLT/UVES (Ultraviolet and Visual Echelle Spectrograph) data of low-column density structures in the Milky Way halo. We detected Ca II $\lambda \lambda 3934.8,3969.6$ (and in some cases also $\mathrm{Na}$ I $\lambda \lambda 5891.6,5897.6)$ absorption features at low, intermediate, and high radial velocities along 35 sight lines (out of a sample of 103) towards high-redshift quasars (QSOs). For several of these sight lines counterparts in $21-\mathrm{cm} \mathrm{H} \mathrm{I} \mathrm{emis-}$ sion were found using the 100-m radio telescope in Effelsberg.

In this work we concentrate on the follow-up study of $\mathrm{HI}$ 21-cm small- scale structures in directions that were previously observed with UVES and the Effelsberg telescope. Richter et al. (2005) observed one sight line (PKS 1448-232) with particularly prominent high-velocity $\mathrm{Ca}$ II and $\mathrm{NaI}$ absorption lines with UVES, Effelsberg, and the VLA. The VLA H I map resolves the HVC into several compact, cold clumps. Here, we present new high-resolution $\mathrm{H}$ I data of four other absorbing systems out of the sample of 35 sight lines. Using these observation makes it possible to estimate important physical and chemical properties, e.g., lower distance and pressure limits, as well as $\mathrm{Ca}$ II and $\mathrm{Na}$ I abundances.

Our paper is organised as follows. In Sect. 2 we describe the data acquisition and reduction. A presentation of the four sight lines observed with VLA and WSRT is given in Sect. 3. In Sect. 4, results regarding the properties of the observed smallscale structures are discussed. We conclude our study in Sect. 5.

\section{Data acquisition and reduction}

A detailed description of our UVES data and the initial H I 21-cm observations with the 100-m telescope at Effelsberg is provided in Ben Bekhti et al. (2008).

For four sight lines (QSO J0003-2323, QSO B1331+170, QSO B0450-1310, and J081331+254503) additional highresolution data were obtained with the Very Large Array (VLA) and the Westerbork Synthesis Radio Telescope (WSRT). These data were used to search for small-scale structure within the diffuse gas clouds. Detailed information about the observations with both telescopes is provided in Table 1, showing the instrument used, the date of observation, the chosen configuration, the coordinates of the sources, field of view $\left(\Omega_{\mathrm{HPBW}}\right)$, the spatial $\left(\Theta_{\text {HPBW }}\right)$ and instrumental spectral resolution $(\delta v)$, the bandwidth $(\Delta v)$, and the resulting noise level $\left(\sigma_{\text {rms }}\right)$ in the final datacubes.

\subsection{Westerbork synthesis radio telscope (WSRT)}

The fourteen $25-\mathrm{m}$ antennas of the WSRT were used in the $2 \times 48$ configuration, providing a good compromise between sensitivity and resolution. For our observations a correlator bandwidth of $2.5 \mathrm{MHz}$ was chosen. The spectral channel width is $0.5 \mathrm{~km} \mathrm{~s}^{-1}$ with a total of 1024 channels. The observing time for both clouds was $12 \mathrm{~h}$ and additionally $1 \mathrm{~h}$ for the (primary) pre- and postcalibrators.

The WSRT data were analysed using the MIRIAD software package. The first step was to flag bad data points in the source and calibrator datasets, in order to exclude these during the following reduction tasks. The primary calibrators were used for the absolute flux, phase, gain, and bandpass calibration. Due to phase changes during the $12 \mathrm{~h}$ observation, an additional relative phase calibration was necessary. For this purpose self-calibration was performed. Finally, dirty image data cubes were calculated via Fast Fourier Transformation (FFT) using a robust parameter of $r=0.5$. The cubes were gridded such that they have a velocity width of $0.5 \mathrm{~km} \mathrm{~s}^{-1}$ per spectral channel. The CLEAN algorithm (Högbom 1974) was applied to reconstruct the image. The resulting data cubes and moment-0 maps were primary- beamcorrected.

\subsection{Very large array (VLA)}

The VLA observations were carried out using the twenty-nine 25-m radio antennas in the DnC configuration. This hybrid configuration consists of the southeastern and southwestern arms in the smallest (D) configuration and the northern arm in the larger $\mathrm{C}$ configuration. It is especially well-suited to extended sources with a declination south of $\delta=-15^{\circ}$. For each of the two polarisations, the correlator provides a bandwidth of $1.56 \mathrm{MHz}$ with 256 spectral channels resulting in a channel separation of $1.3 \mathrm{~km} \mathrm{~s}^{-1}$.

The observing time for the sightline in the direction of QSO J0003-2323 was $6 \mathrm{~h}$ and $12 \mathrm{~h}$ for QSO B1331+170 with additional $1 \mathrm{~h}$ allocated for the primary and secondary calibrators. The primary calibrators $0137+331$ and $0542+498$ were observed for about $30 \mathrm{~min}$ each at the beginning and the end of the observation periods. The secondary calibrators $0025-260$ and 0447-220 were observed once every hour for about $5 \mathrm{~min}$.

The VLA data of QSO B0450-1310B and QSO J0003-2333 were contaminated by many interferences and antenna failures. As both sources were observed on multiple occasions, the individual parts had to be handled separately and were only merged after self-calibration. The data reduction was performed with MIRIAD. First, a calibration based on the observed primary and secondary calibrators was done. A median filter was applied to the visibilities per baseline to remove outliers, and three malfunctioning antennas as well as obvious interferences were completely flagged. Along with continuum subtraction, an iterative self-calibration was carried out to account for phase changes during the observations. After merging the respective visibility 

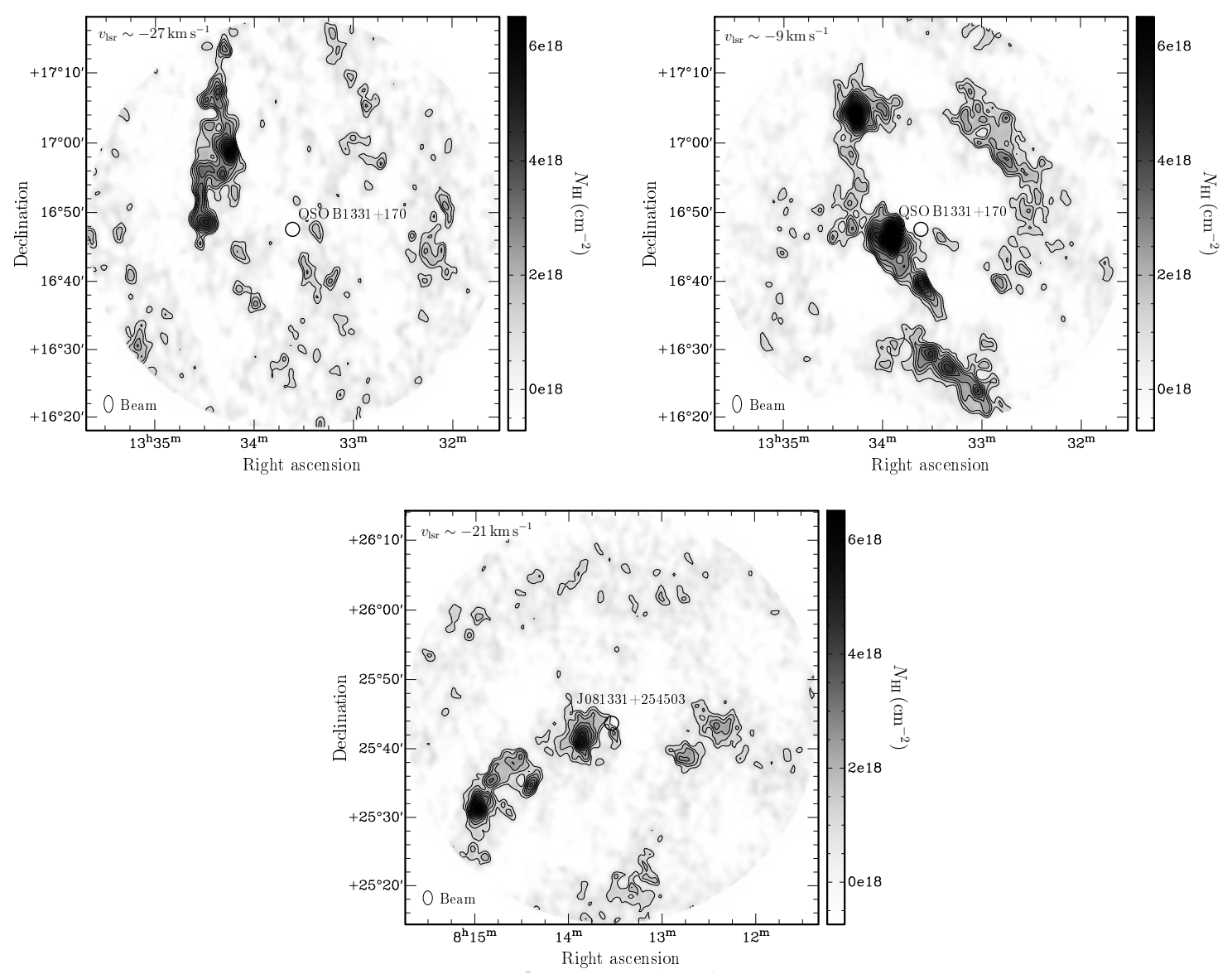

Fig. 1. Upper panels: WSRT $21-\mathrm{cm}$ column density maps of the intermediate-velocity gas in the direction of the quasar QSO B1331+170. Several small H I clumps with low column densities $\left(N_{\mathrm{HI}} \leq 1.6 \times 10^{19} \mathrm{~cm}^{-2}\right)$ are detected at two distinct velocities of $v_{\text {lsr }} \approx-27$ and $v_{\text {lsr }} \approx-10 \mathrm{~km} \mathrm{~s}$ (column densities are calculated using the velocity interval $v_{\mathrm{lsr}}=-30 \ldots-21 \mathrm{~km} \mathrm{~s}^{-1}$ and $v_{\mathrm{lsr}}=-14 \ldots-1 \mathrm{~km} \mathrm{~s}^{-1}$, respectively). The beam size of $80^{\prime \prime} \times 150^{\prime \prime}$ is shown in the maps. The contours start at $2 \sigma_{\mathrm{rms}}$ in steps of $\sigma_{\mathrm{rms}}=5 \times 10^{17} \mathrm{~cm}^{-2}$. The size of the synthesised beam is shown in the lower left corner. Lower panel: WSRT 21-cm map (integrated over $v_{\mathrm{lsr}}=-25 \ldots-18 \mathrm{~km} \mathrm{~s}^{-1}$ ) of the intermediate-velocity gas in the direction of the quasar J081331+254503. Several small H I clumps with low column densities $\left(N_{\mathrm{HI}} \leq 2 \times 10^{19} \mathrm{~cm}^{-2}\right)$ are detected. The beam size of $80^{\prime \prime} \times 120^{\prime \prime}$ is shown in the figure. The contours start at $2 \sigma_{\text {rms }}$ in steps of $\sigma_{\mathrm{rms}}=5 \times 10^{17} \mathrm{~cm}^{-2}$. Note that the maps were not primary-beam corrected to improve visualisation.

sets, data cubes were calculated via FFT using a robust parameter of $r=0.4$ for QSO J0003-2333 and of $r=2$ for QSO B0450-1310B. To improve sensitivity, the beam was artificially increased to a size of $100^{\prime \prime} \times 100^{\prime \prime}$ by convolution with a Gaussian. The resulting data cubes have a velocity width of $1.3 \mathrm{~km} \mathrm{~s}^{-1}$ per spectral channel. Again, primary beam corrections were applied.

In Fig. 1 we show the HI (integrated) column densities of the QSO sightlines QSO B1331+170 and J081331+254503 associated to our absorbers. Figure 2 contains the maps for QSO J0003-2323 and QSO B0450-1310. The velocity ranges over which was integrated are given in the figure captions. The spectral widths of the sources are small, so we decided not to show position-velocity plots (or individual spectral maps). We clipped the maps for radii over 0.5 degrees (which is the HPBW of the primary beam of the WSRT and VLA). To improve visualisation, the maps shown in Figs. 1 and 2 were not corrected for the primary beam. Hence, they do not show true flux values. The clumps that have been analysed are presented in a subsequent section with primary beam correction applied. In the case of the VLA observations, residual image artifacts (stripes) are visible in the maps. We carefully inspected the data cubes in adjacent (empty) velocity planes. There, none of these stripes are present. It might be that the H I sources partially absorb the QSO continuum leading to baseline fitting problems. However, the clumps are detected with much higher significance than could be caused by the artificial emission. Nevertheless, the total column density of the clumps in the direction of QSO J0003-2323 and QSO B0450-1310 may be overestimated by a few percent.

The datacubes were also inspected for emission at other radial velocities than for the absorbers. In the case of $\mathrm{J} 081331+254503$ we found few very weak $\left(N_{\mathrm{HI}}<5 \times\right.$ $10^{18} \mathrm{~cm}^{-2}$ ) small clumps at $v_{\mathrm{lsr}} \sim-60 \mathrm{~km} \mathrm{~s}^{-1}$. It was not analysed more, because we are interested here only in the correlation between emission and absorption. The other sight lines do not reveal additional sources.

In the next section we present the results of the physical analysis of the clumps that were significantly detected.

\section{The selected sight lines}

Inspired by the results of Richter et al. (2005) and Ben Bekhti et al. (2008), we observed four sightlines (out of a sample of 35) again that exhibit prominent $\mathrm{Ca}$ II and/or $\mathrm{Na}$ I halo absorption features with high-resolution radio interferometers. The aim of this study is to search for those small-scale structures embedded in the neutral hydrogen gas that were possibly not resolved with the single-dish Effelsberg telescope. 

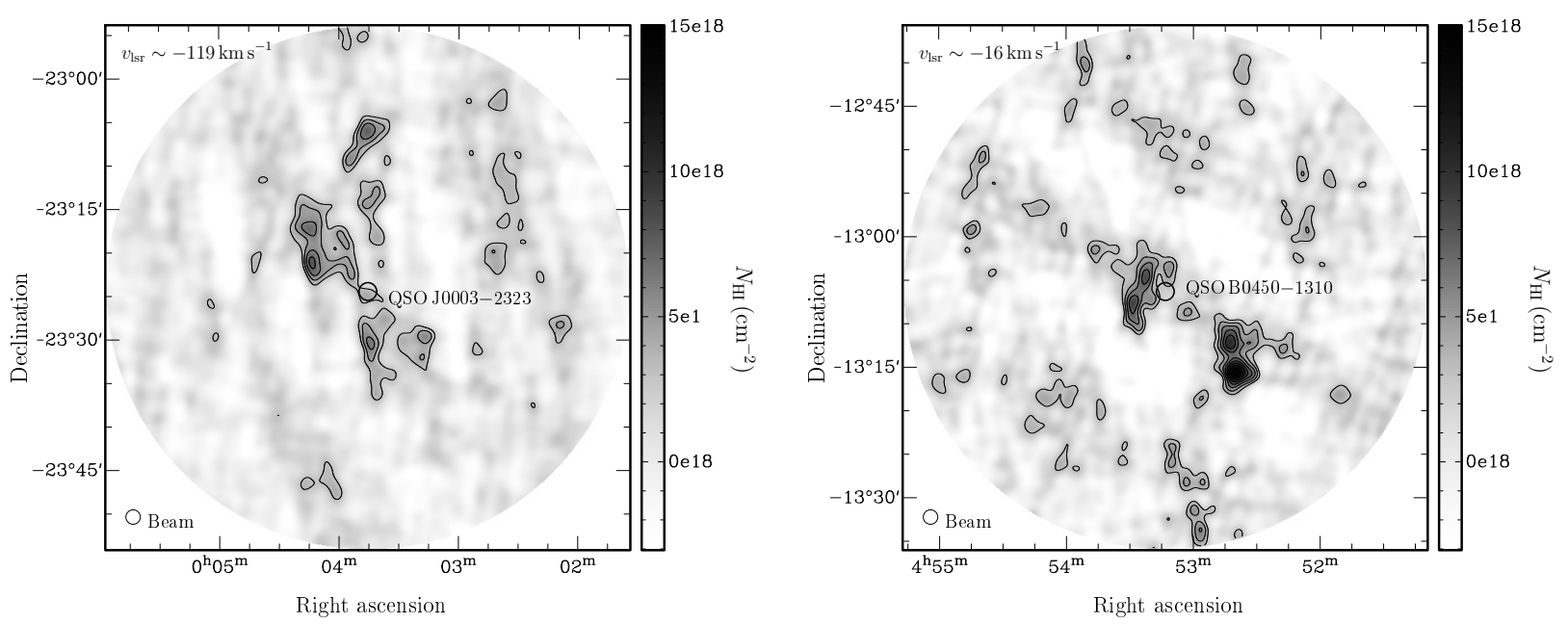

Fig. 2. Left panel: VLA $21-\mathrm{cm}$ column density map (integrated over $v_{\mathrm{lsr}}=-121 \ldots-116 \mathrm{~km} \mathrm{~s}^{-1}$ ) of the high-velocity gas in the direction of the quasar QSO J0003-2323. Several H I clumps with low column densities $\left(N_{\mathrm{HI}} \leq 1.9 \times 10^{19} \mathrm{~cm}^{-2}\right)$ are detected. The beam size of $100^{\prime \prime} \times 100^{\prime \prime}$ is indicated in the lower right corner. The contours start at $2 \sigma_{\mathrm{rms}}$ in steps of $\sigma_{\mathrm{rms}}=1.5 \times 10^{18} \mathrm{~cm}^{-2}$. Right panel: VLA 21-cm column density map (integrated over $v_{\mathrm{lsr}}=-22 \ldots-9 \mathrm{~km} \mathrm{~s}^{-1}$ ) of the low-velocity gas observed along the sight line towards QSO B0450-1310. H I clumps with column densities $N_{\mathrm{HI}} \leq 2.3 \times 10^{19} \mathrm{~cm}^{-2}$ are detected. The beam size of $100^{\prime \prime} \times 100^{\prime \prime}$ is shown in the lower left corner. The contours start at $2 \sigma_{\text {rms }}$ in steps of $\sigma_{\mathrm{rms}}=1.5 \times 10^{18} \mathrm{~cm}^{-2}$. Note that the maps were not primary-beam corrected to improve visualisation.

Table 2. Summary of UVES Ca II, Na I and Effelsberg H I measurements towards the four quasars.

\begin{tabular}{|c|c|c|c|c|c|c|c|c|c|c|}
\hline Quasar & $\begin{array}{c}l \\
{\left[{ }^{\circ}\right]}\end{array}$ & $\begin{array}{c}b \\
{\left[{ }^{\circ}\right]} \\
\end{array}$ & $\begin{array}{c}v_{\text {LSR }} \\
{\left[\mathrm{km} \mathrm{s}^{-1}\right]} \\
\end{array}$ & $\begin{array}{c}\log N_{\text {CaII }} \\
{\left[N \text { in } \mathrm{cm}^{-2}\right]}\end{array}$ & $\begin{array}{c}b_{\text {CaII }} \\
{\left[\mathrm{km} \mathrm{s}^{-1}\right]}\end{array}$ & $\begin{array}{c}\log N_{\mathrm{NaI}} \\
{\left[N \text { in cm } \mathrm{cm}^{-2}\right]}\end{array}$ & $\begin{array}{c}b_{\mathrm{NaI}} \\
{\left[\mathrm{km} \mathrm{s}^{-1}\right]} \\
\end{array}$ & $\begin{array}{c}\log N_{\mathrm{HI}} \\
{\left[N \mathrm{in} \mathrm{cm}^{-2}\right]}\end{array}$ & $\begin{array}{c}b_{\mathrm{HI}} \\
{\left[\mathrm{km} \mathrm{s}^{-1}\right]}\end{array}$ & $\begin{array}{l}\text { HVC/IVC } \\
\text { complex }\end{array}$ \\
\hline \multirow[t]{3}{*}{ QSO J0003-2323 } & 49.4 & -78.6 & -98 & 11.9 & 6 & - & - & - & - & MS \\
\hline & & & -112 & 11.8 & 6 & - & - & 19.6 & 19.3 & MS \\
\hline & & & -126 & 11.9 & 6 & - & - & - & - & MS \\
\hline \multirow[t]{2}{*}{ QSO B1331+170 } & 348.5 & 75.8 & -9 & 12.4 & 11 & 11.3 & 6 & 18.9 & 2.7 & - \\
\hline & & & -27 & 12.0 & 5 & 11.3 & 3 & - & - & IV Spur \\
\hline \multirow[t]{2}{*}{ QSO B0450-1310B } & 211.8 & -32.1 & -5 & - & - & 11.5 & 5 & 19.8 & 2.3 & - \\
\hline & & & -20 & - & - & 12.0 & 2 & 19.3 & 6.8 & - \\
\hline $\mathrm{J} 081331+254503$ & 196.9 & 28.6 & -23 & - & - & 11.1 & 5 & 19.8 & 37.0 & (LLIV Arch) \\
\hline
\end{tabular}

In this section, the results of the high-resolution radio observations of the four Ca II and Na II halo absorbers are presented. We also summarise briefly the results of the analysis of the Ca II and Na I (UVES) and low- resolution H I (Effelsberg) data. Voigt profiles were fitted to the absorption lines, and Gaussians were used to parametrise the emission lines. Table 2 lists the position, LSR velocities, Ca II, Na I, and H I column densities, Doppler parameters $b$, and the possibly associated IVC or HVC complexes. All of the UVES and Effelsberg spectra shown here are plotted in the local standard-of-rest (LSR) velocity frame. Most of the gas in emission and absorption near zero velocities belongs to the local Galactic disk. As described in Ben Bekhti et al. (2008), the decision whether the gas is connected to the Galactic disk or halo is based on a Milky Way model developed by Kalberla (2003) and Kalberla et al. (2007). The model predicts for each direction the expected velocities for interstellar gas participating in Galactic disk rotation. This means that the deviation velocity (Wakker 1991) can be calculated and is used to classify the sources as IVCs or HVCs. The $4 \sigma$ and $2 \sigma$ detection limits for the Ca II $\lambda 3934.77$ and Ca II $\lambda 3969.59$ lines, respectively, correspond to a $\log \left(N_{\mathrm{CaII}} / \mathrm{cm}^{-2}\right)$ detection limit of $\approx 11$. The parameters of all detected absorption and emission components for 35 sight lines are listed in Ben Bekhti et al. (2008).

Using the reduced high-resolution data cubes and the resulting column density maps, various physical parameters were derived. All structures above a threshold of $4 \sigma_{\mathrm{rms}}$ (in the integrated column density maps) were considered in our analysis. From the moment-0 maps, the peak column densities were inferred, as well as the angular sizes of individual clumps (using the Gaussian radial fit method of the Karma task kvis). The latter, however, can only be considered rough estimates, as the clumps are neither necessarily spherical nor must they have Gaussian column density profiles. The spectra containing the highest total flux were extracted, and Gaussian velocity profiles were fitted. This returned peak fluxes (which can be converted to peak brightness temperatures, $T_{\mathrm{B}}$ ), velocity widths (leading to an upper temperature limit), and LSR velocities of each clump. Velocity profile widths are not only given in FWHM but are also converted to $b$-values to allow for better comparison with the absorption lines. The derived physical properties of the clumps are summarised in Table 3, containing the clump IDs, the coordinates, the velocities in the LSR frame, the FWHM derived from a Gauss fit and associated $b$-values, the peak amplitudes, the peak H I column densities (with a typical error of about $10^{18} \mathrm{~cm}^{-2}$ ), the peak brightness temperatures, the upper temperature limits, and the mean radial sizes, $\phi$ (FWHM).

\subsection{QSO J0003-2323}

The upper panel of Fig. 3 shows the multi-component Ca II halo absorbers in the direction of QSO J0003-2323 as observed with UVES. Ca II $\lambda \lambda$ 3934.77, 3969.59 absorption at high velocities 
Table 3. Physical properties of the H I clumps in the direction of the quasars J081331+254503, QSO B1331+170 (observed with the WSRT), QSO J0003-2323, and QSO B0450-1310B (observed with the VLA).

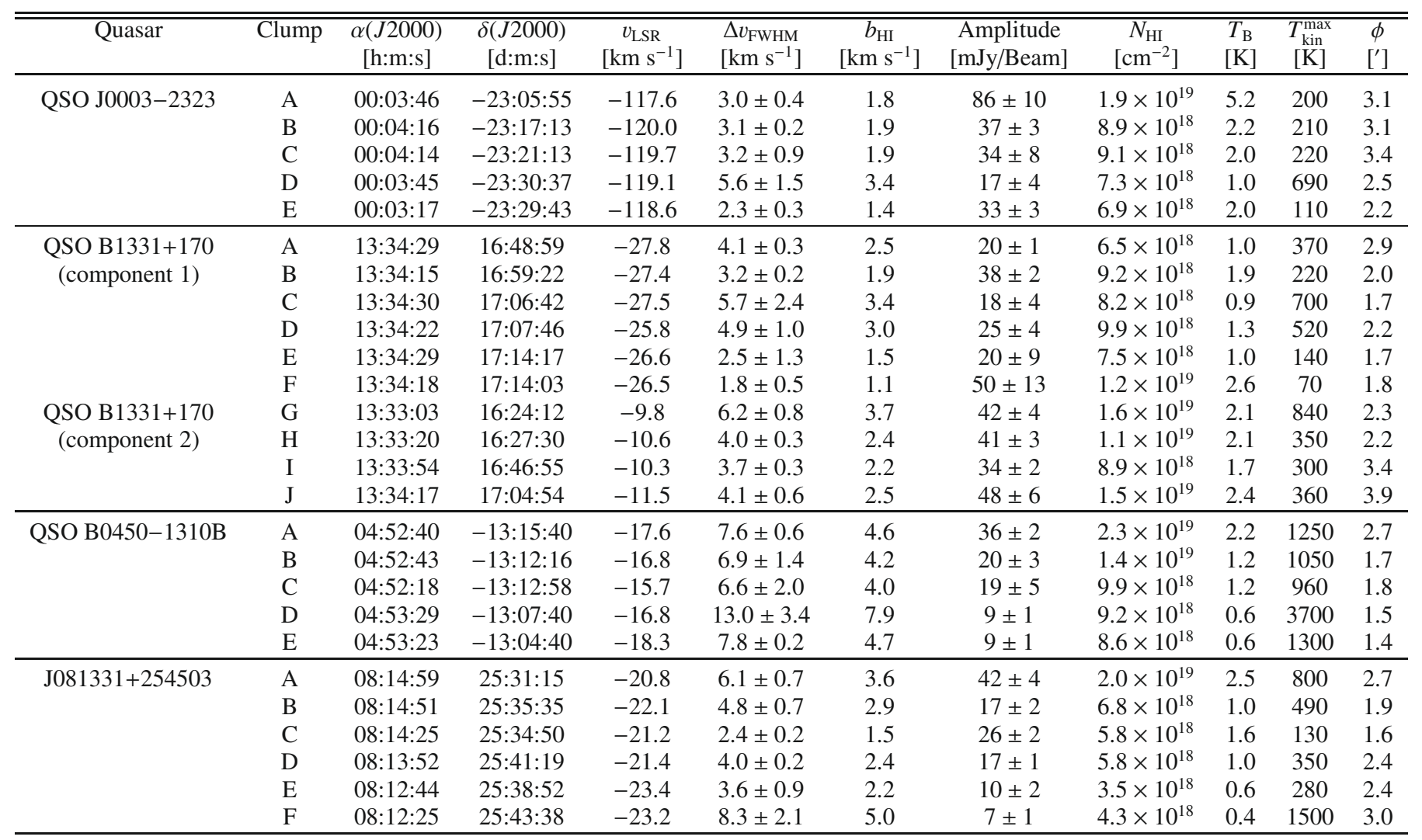

is detected with high significance $\left(31 \sigma_{\text {rms }}\right)$ level, but no corresponding $\mathrm{Na}$ I is seen. The Ca II absorption spreads between $v_{\text {lsr }} \approx-120 \ldots-80 \mathrm{~km} \mathrm{~s}^{-1}$ having $b_{\text {CaII }} \approx 6 \mathrm{~km} \mathrm{~s}^{-1}$. A corresponding broad $\left(b=19.3 \mathrm{~km} \mathrm{~s}^{-1}\right) 21-\mathrm{cm}$ emission line is found in the Effelsberg data at $v_{\text {lsr }} \approx-112 \mathrm{~km} \mathrm{~s}^{-1}$ with an H I column density of about $\log \left(N_{\mathrm{HI}} / \mathrm{cm}^{-2}\right)=19.6$ (see upper panel of Fig. 1). This absorbing system is kinematically compact; i.e., multiple components with comparable $b$-values are spread over less than $\approx 100 \mathrm{~km} \mathrm{~s}^{-1}$ (Ding et al. 2005). The position and the velocities of this $\mathrm{Ca}$ II absorber indicate a possible association with the Magellanic Stream (Ben Bekhti et al. 2008).

The lower panel of Figure 3 shows the high-resolution $\mathrm{HI}_{\mathrm{I}}$ column density map of the high-velocity gas in the direction of QSO J0003-2323 integrated over $v_{\mathrm{lsr}}=-121 \ldots-116 \mathrm{~km} \mathrm{~s}^{-1}$, as observed with the VLA. The observations reveal the presence of several H I clumps. Clumps with $\geq 4 \sigma_{\text {rms }}\left(\sigma_{\text {rms }}=1.6 \times\right.$ $10^{18} \mathrm{~cm}^{-2}$ ) significance level are labelled with letters A to $\mathrm{E}$.

The small-scale clumps have H I peak column densities of $\approx 7 \ldots 9 \times 10^{18} \mathrm{~cm}^{-2}$, except for clump A, which has $1.9 \times$ $10^{19} \mathrm{~cm}^{-2}$, corresponding to peak brightness temperatures of $T_{\mathrm{B}} \approx 1 \ldots 2 \mathrm{~K}$ (clump A: $5 \mathrm{~K}$ ). All clumps have angular diameters of $\phi<3.5^{\prime}$ (FWHM) and are mostly relatively isolated or only weakly connected to each other. However, this could be an instrumental effect as the interferometric data lack small spatial frequencies. Note also that the clumps might not be resolved by the VLA beam, as the spatial features visible in the map approximately match the size of the beam $\left(100^{\prime \prime} \times 100^{\prime \prime}\right)$. This means that there could be even more substructure within the cores.

The line widths are typically very small with $\Delta v_{\text {FWHM }} \leq$ $3 \mathrm{~km} \mathrm{~s}^{-1}$, implying that the absorbing gas is cool with an upper temperature limit of $T_{\text {kin }} \approx 200 \mathrm{~K}$. Only clump D reveals slightly larger velocity widths, leading to an upper limit of $T_{\text {kin }} \leq 700 \mathrm{~K}$. An important point is that only one emission line was detected in the H I single dish and interferometric data, while the absorption measurements reveal a multi-component structure. A slight velocity offset also exists between the Ca II absorption components and the velocities of the H I clumps seen in emission (see Tables 2 and 3). This indicates that absorption and emission measurements trace somewhat different regions of the same overall structure. Figure 1 (lower panel) shows that the quasar sightline does not pass any of the clumps directly, but instead traces the outer boundary of clump $\mathrm{C}$, which is barely detected in 21-cm emission. The detection of multiple, relatively strong Ca II absorption components towards QSO J0003-2323 thus indicates that there are additional, low-column density neutral gas structures along this sightlines that cannot be detected and/or resolved even with deep $21-\mathrm{cm}$ synthesis observations. The complex spatial structure and kinematics of the outer layers of HVCs have been previously observed (Brüns 1998; Brüns et al. 2001; Ben Bekhti et al. 2006) and were also found in numerical simulations (Quilis \& Moore 2001), pointing towards interactions of the clouds with the ambient medium, i.e., ram- pressure causing head-tail structures.

\subsection{QSO B1331+170}

The intermediate-velocity CaII absorber towards QSO B1331+170 is shown in the upper left panel of Figure 4. This two-component system is detected in Ca II (at $19 \sigma$ significance), as well as in Na I. The lines spread between $v_{\mathrm{lsr}} \approx-40 \ldots 0 \mathrm{~km} \mathrm{~s}^{-1}$. The position and velocity of the line 

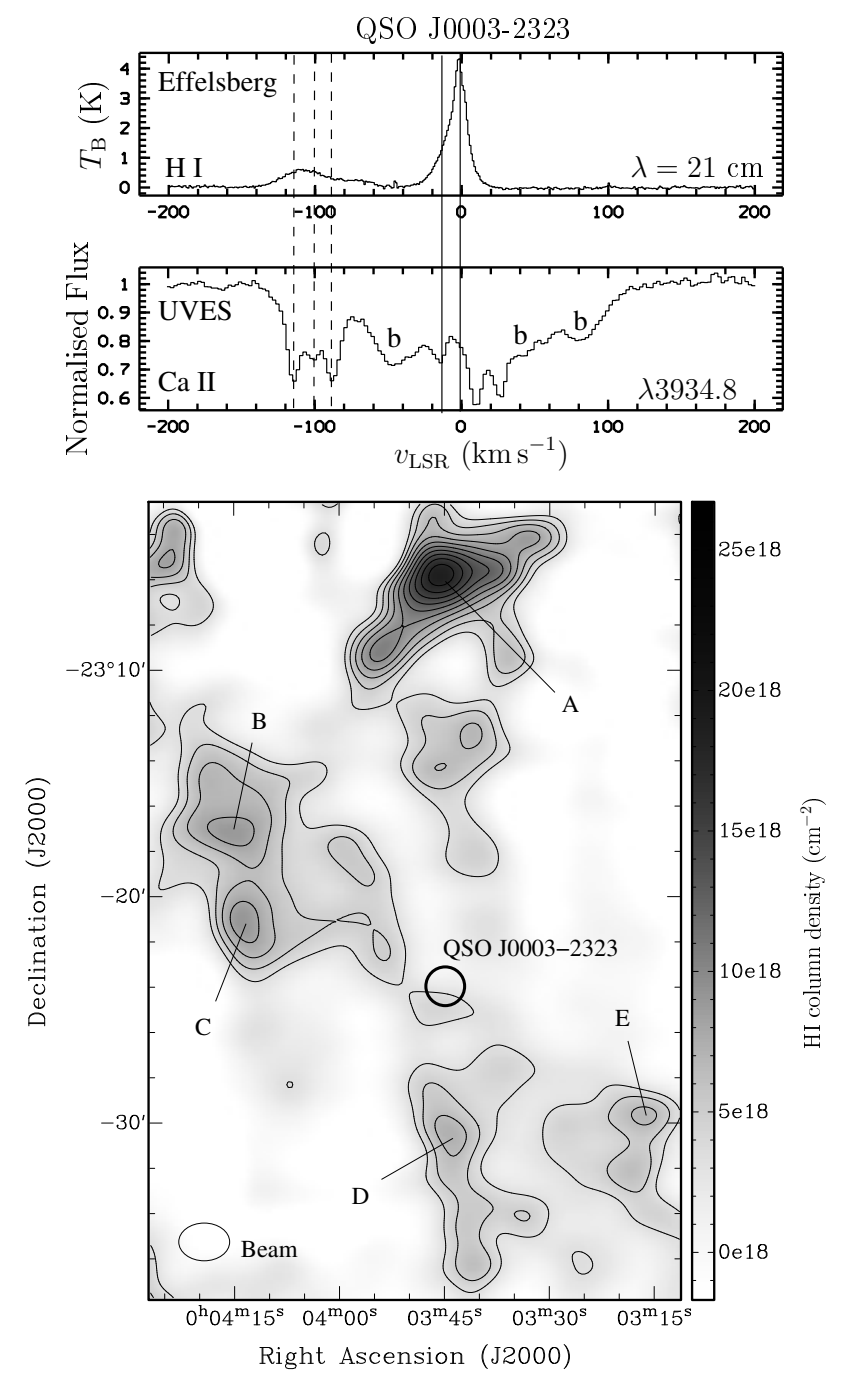

Fig. 3. Upper panel: CaII $\lambda 3934.8$ absorption profile towards QSO J0003-2323. Additionally, the corresponding emission profile of H I $21-\mathrm{cm}$ is shown as observed with the 100-m telescope at Effelsberg. The dashed vertical lines indicate the absorption components which were identified. The solid lines mark the minimal and maximal LSR velocities which are expected for the Galactic disk gas in this direction according to the Milky Way model developed by Kalberla (2003); Kalberla et al. (2007); b denotes blending lines from intervening absorption systems. Lower panel: VLA $21-\mathrm{cm}$ column density map showing the clumps A to E in the direction of the quasar QSO J0003-2323. The contour lines are identical to those in Fig. 2 (left panel).

at $v_{\mathrm{lsr}} \approx-27 \mathrm{~km} \mathrm{~s}^{-1}$ indicate a possible association with the intermediate-velocity complex IV Spur.

There is a corresponding $\mathrm{HI}$ line at $v_{\mathrm{lsr}} \approx-9 \mathrm{~km} \mathrm{~s}^{-1}$ detected with the 100-m telescope at Effelsberg and possibly also a feature at $-27 \mathrm{~km} \mathrm{~s}^{-1}$, but not detected clearly. The peak column density of the observed line is $N_{\mathrm{HI}}=6.3 \times 10^{19} \mathrm{~cm}^{-2}$.

The high-resolution WSRT $21-\mathrm{cm}$ column density map (integrated over $v_{\mathrm{lsr}}=-30 \ldots-21 \mathrm{~km} \mathrm{~s}^{-1}$ and $v_{\mathrm{lsr}}=$ $-14 \ldots-1 \mathrm{~km} \mathrm{~s}^{-1}$, respectively) reveals two components at $v_{\mathrm{lsr}} \approx$ $-27 \mathrm{~km} \mathrm{~s}^{-1}$ (component 1 ) and $v_{\mathrm{lsr}} \approx-10 \mathrm{~km} \mathrm{~s}^{-1}$ (component 2 ), nicely matching the absorption signatures in velocity. In the first case, six clumps are identified and labelled with letters $\mathrm{A}$ to $\mathrm{F}$. The $\mathrm{H}$ I peak column densities range from about $\approx 6.5 \times 10^{18} \mathrm{~cm}^{-2}$ to $1.2 \times 10^{19} \mathrm{~cm}^{-2}$ and have peak brightness temperatures of the order of $T_{\mathrm{B}} \approx 1.0 \ldots 2.5 \mathrm{~K}$. Compared to the other three sight lines and component 2 , this structure is spatially more compact. Furthermore, the individual clumps are less isolated. For component 1 the spatial separation between the position of the quasar and the high-resolution emission is about $10^{\prime}$. The angular diameter $\phi$ of the clumps is about $2^{\prime}$. The line widths of the six clumps are in the range of $\Delta v_{\mathrm{FWHM}} \approx 2 \ldots 6 \mathrm{~km} \mathrm{~s}^{-1}$, which implies that the absorbing gas has a temperature of $T_{\mathrm{kin}}^{\max } \leq 700 \mathrm{~K}$. Clump F has an extraordinarily small line width of only $1.8 \mathrm{~km} \mathrm{~s}^{-1}\left(T_{\text {kin }}^{\max } \leq 70 \mathrm{~K}\right)$. Component 2 exhibits four clumps ( $\mathrm{G}$ to $\mathrm{J}$ ) with higher column densities of $\approx 0.9 \ldots 1.6 \times 10^{19} \mathrm{~cm}^{-2}$ and peak brightness temperatures $(\approx 2 \mathrm{~K})$.

To check whether there is a physical connection between both components, we computed an integrated position-velocity map; see Fig. 5. There is a faint emission feature connecting clumps B and $\mathrm{J}$; however, the feature is close to the noise level $\left(4 \sigma_{\text {rms }}\right)$. Possibly, both components stem from an outflow, with component 2 very close (in velocity) to the Milky Way emission. Component 1 might just represent a feature that has been expelled more recently (hence having higher velocity).

\subsection{QSO B0450-1310}

Figure 6 shows the two-component absorber at low and intermediate velocities towards QSO B0450-1310. Two Na I lines $(\lambda \lambda$ 5891.58,5897.56) were clearly detected at a $25 \sigma$ and a $11 \sigma$ level, respectively. Unfortunately, no Ca II data are currently available for this line of sight. The two absorption lines have central velocities of $v_{\text {lsr }} \approx-5$ and $-20 \mathrm{~km} \mathrm{~s}^{-1}$. There is no obvious association with any known IVC complex.

For both components, H I counterparts in the spectrum obtained with the 100-m telescope at Effelsberg were found, with low $b_{\mathrm{HI}}$-values of 2.3 and $6.8 \mathrm{~km} \mathrm{~s}^{-1}$. Such narrow line widths were also measured for the $\mathrm{Na}$ I absorption lines $\left(b_{\mathrm{NaI}}=\right.$ $\left.2.5 \mathrm{~km} \mathrm{~s}^{-1}\right)$. The column densities of both H I systems are relatively low with $\log \left(N_{\mathrm{HI}} / \mathrm{cm}^{-2}\right)=19.8$ and $\log \left(N_{\mathrm{HI}} / \mathrm{cm}^{-2}\right)=$ 19.3. The Na I absorption line at $-20 \mathrm{~km} \mathrm{~s}^{-1}$ reveals a higher column density and smaller width than the line at $-5 \mathrm{~km} \mathrm{~s}^{-1}$. Interestingly, this is the opposite of what is observed for the H I emission.

The H I high-resolution column density map (integrated over $v_{\text {lsr }}=-22 \ldots-9 \mathrm{~km} \mathrm{~s}^{-1}$ ) obtained with the VLA displays two main structures consisting of few clumps each. Clumps with a significance level of $\geq 4 \sigma_{\mathrm{rms}}\left(\sigma_{\mathrm{rms}}=1.6 \times 10^{18} \mathrm{~cm}^{-2}\right)$ were labelled with letters $\mathrm{A}$ to $\mathrm{E}$. The peak brightness temperatures are in the range of $T_{\mathrm{B}}=0.6 \ldots 2.2 \mathrm{~K}$, corresponding to H I column densities between $9 \times 10^{18} \mathrm{~cm}^{-2}$ and $2.3 \times 10^{19} \mathrm{~cm}^{-2}$. The angular diameters are very small with $\phi \approx 1.5 \ldots 2.5^{\prime}$. The line widths of the clumps vary from about $\Delta v_{\mathrm{FWHM}}=7 \mathrm{~km} \mathrm{~s}^{-1}$ up to $\Delta v_{\text {FWHM }}=13 \mathrm{~km} \mathrm{~s}^{-1}$, which correspond to upper temperature limits of $T_{\text {kin }} \approx 1000 \mathrm{~K}$ and $T_{\text {kin }} \approx 3700 \mathrm{~K}$ and is much higher than derived for the other sight lines. The small-scale features lie at velocities of $v_{\text {lsr }} \approx-18 \mathrm{~km} \mathrm{~s}^{-1}$, which is close to the higher-column density absorption line, while the diffuse gas observed with the 100-m telescope is mainly found at different velocities. This shows again that absorption and emission measurements sample somewhat different regions and gas phases within the overall cloud complex. Possibly, the gas interacts with an ambient medium, producing colder cores containing $\mathrm{Na}$ I in the interface region, while the diffuse gas is dragged away by ram pressure. 

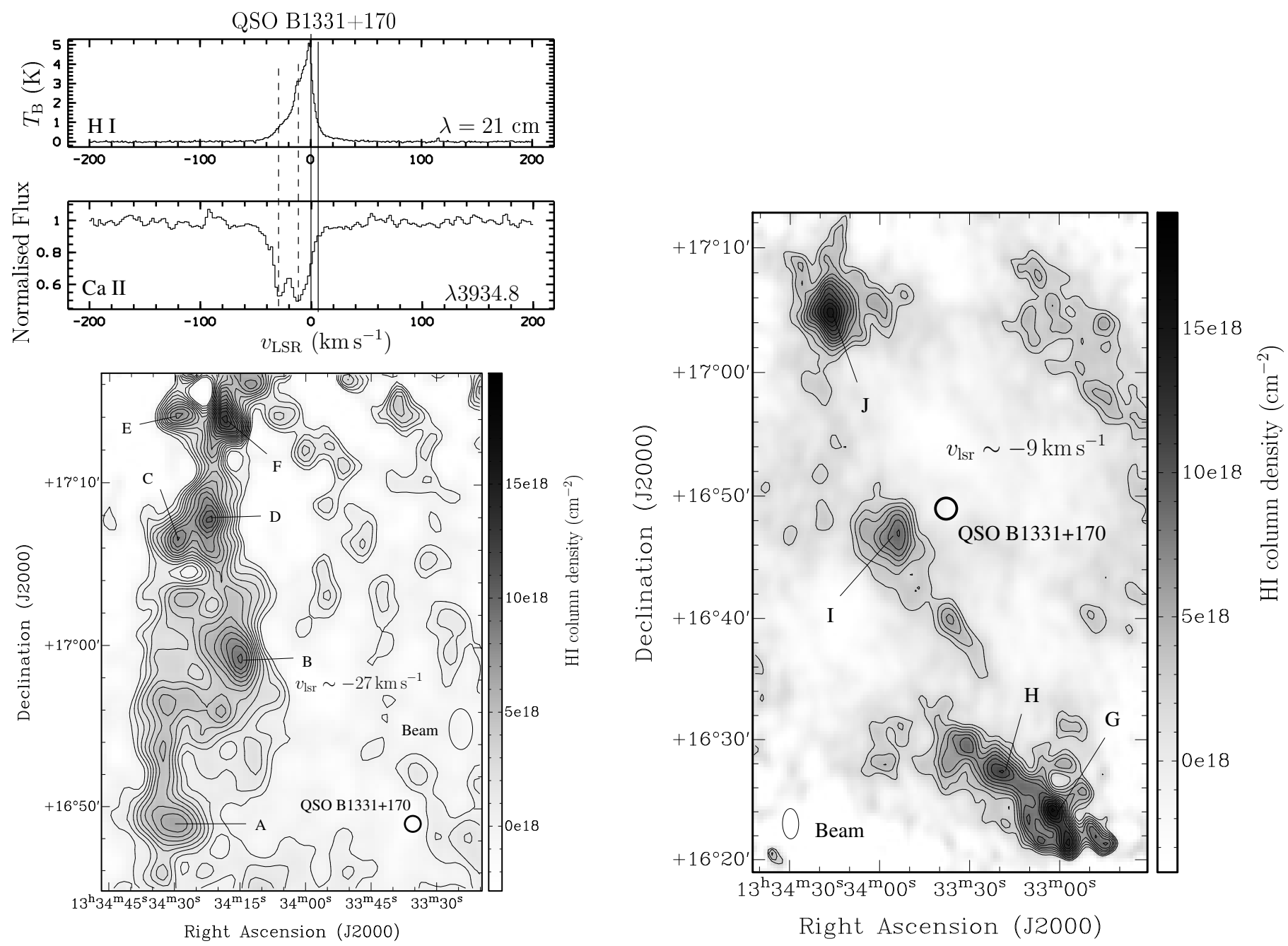

Fig. 4. Upper left panel: Ca II absorption and corresponding H I emission spectra towards QSO B1331+170. Lower left and right panel: WSRT $21-\mathrm{cm}$ column density maps of the intermediate-velocity gas in the direction of the quasar QSO B1331+170. Note, that for the component at $v_{\mathrm{lsr}} \approx-27 \mathrm{~km} \mathrm{~s}^{-1}$ the line of sight towards QSO B1331+170 lies about 10' away from the nearest clump, while for the second component the line of sight is located near clump I. The contour lines are identical to those in Fig. 1 (upper panels).

\section{4. $J 081331+254503$}

The only single-component absorber in our absorber sample lies in the direction of the quasar J081331+254503 (see Fig. 7). Na I $\lambda \lambda 5891.58,5897.56$ absorption is detected at $v_{\mathrm{lsr}} \approx-21 \mathrm{~km} \mathrm{~s}^{-1}$ at a $11 \sigma$ level. There is no Ca II spectrum available for this sight line. The corresponding Effelsberg H I emission line has a $b_{\mathrm{HI}}$-value of about $37.0 \mathrm{~km} \mathrm{~s}^{-1}$ and a column density of $N_{\mathrm{HI}}=6.3 \times 10^{19} \mathrm{~cm}^{-2}$. There is no association with any known IVC complex.

Figure 7 shows the WSRT 21-cm map of the intermediatevelocity gas in the direction of the quasar integrated over $v_{\mathrm{lsr}}=$ $-25 \ldots-18 \mathrm{~km} \mathrm{~s}^{-1}$. The high-resolution observations reveal the presence of six H I clumps labelled with the letters A to F. These small-scale clumps $\left(\phi \leq 3^{\prime}\right)$ have typical peak H I column densities in the range of $\approx 0.4 \ldots 2.0 \times 10^{19} \mathrm{~cm}^{-2}$ and peak brightness temperatures of $T_{\mathrm{B}} \approx 0.5 \ldots 2.5 \mathrm{~K}$. The line widths of $\Delta v_{\mathrm{FWHM}} \leq 8.3 \mathrm{~km} \mathrm{~s}^{-1}$ lead to an upper temperature limit of $T_{\text {kin }} \approx 1500 \mathrm{~K}$. The mean radial velocity is $v_{\text {lsr }} \approx-22 \mathrm{~km} \mathrm{~s}^{-1}$ matching that of the absorption.

It might be possible that the clumps are part of the lowlatitude IV arch (LLIV arch). However, they have slightly lower radial velocities than reported for the LLIV arch (Wakker 2001), so that the association is certain.

\section{Discussion}

As suggested by previous results (Richter et al. 2005), the follow-up observations with the WSRT and the VLA of four quasar sight lines that exhibit low-, intermediate-, and highvelocity $\mathrm{Ca}$ II (Na I) absorption in optical spectra reveal several compact HI clumps that are associated with the absorbers. In the case of the low-velocity sources (QSO B1331+170 component 2 and eventually QSO B0450-1310, both having $v_{\mathrm{dev}}<$ $10 \mathrm{~km} \mathrm{~s}^{-1}$ ), it is unclear whether the sources belong to the Milky Way disk (at somewhat anomalous velocities due to turbulent motions) or tracing the interface region between the halo and the disk. All our clouds have H I column densities below $3 \times 10^{19} \mathrm{~cm}^{-2}$, which is lower than the median value reported by Heiles \& Troland (2003) of $5 \times 10^{19} \mathrm{~cm}^{-2}(\mathrm{CNM})$ for disk clouds in the solar neighbourhood and also lower than the column densities of clouds at high forbidden velocities in the inner galaxy as found by Stil et al. (2006). On the other hand, Ford et al. (2008) find H I clouds in the lower halo dominated by galactic rotation with $N_{\mathrm{HI}} \sim 0.2 \ldots 2.2 \times 10^{19} \mathrm{~cm}^{-2}$, which is similar 


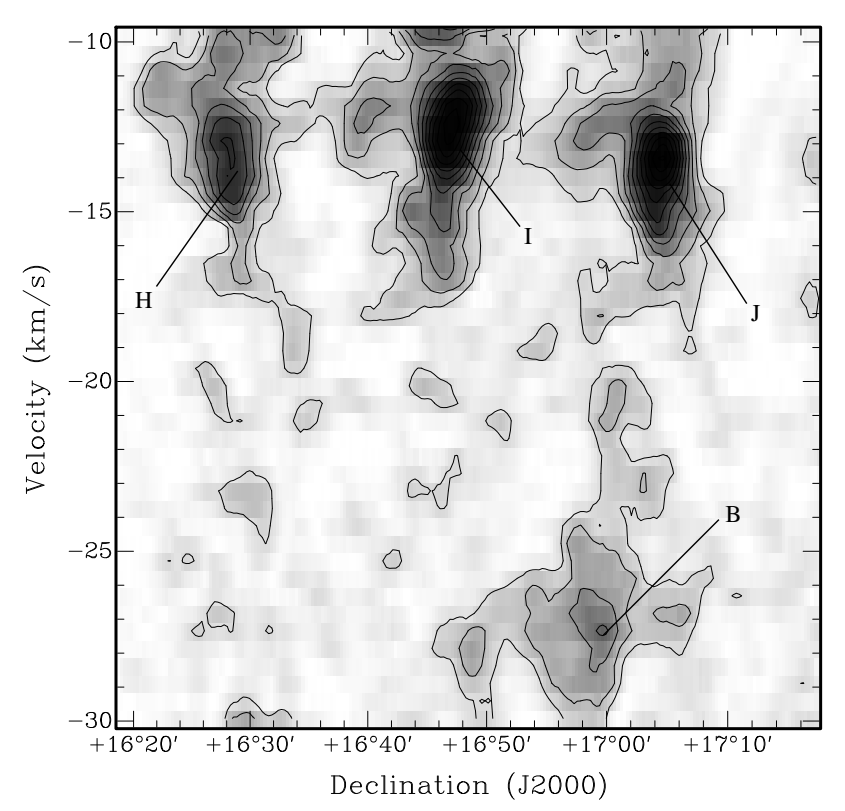

Fig. 5. Position-velocity map of QSO B1331+170 integrated over the range $\mathrm{RA}=\left[13^{\mathrm{h}} 32^{\mathrm{m}} 31^{\mathrm{s}}, 13^{\mathrm{h}} 35^{\mathrm{m}} 38^{\mathrm{s}}\right]$. We did not use the primary-beam corrected cube as the high noise levels towards the edges would degrade the signal-to-noise level in the map significantly. Milky Way emission was not included in the image section. Contours start at $2 \sigma_{\text {rms }}$ in steps of $\sigma_{\text {rms }}$. The map reveals a faint emission feature between clump B (which is part of component 1 ) and clump $\mathbf{J}$ (part of component 2). However, it is not sure, if there is a physical connection or whether this signal is just noise.

to our results. Without knowing the distance to the sources we cannot therefore rule out one of the two possibilities.

In the following, we discuss the physical properties of these clumps.

\subsection{Distance-dependent physical parameters}

The coordinates, as well as the radial velocities of QSO B1331+170 (component 1) and QSO J0003-2323, suggest that the low-column density gas is part of the IVC complex IV Spur and the Magellanic Stream (MS), respectively. The clumps have an angular diameter of $\lesssim 3.5^{\prime}$. Assuming that they are spherical and located at the same distance of about $1 \mathrm{kpc}$ as for IV Spur (Wakker 2001) and about $50 \mathrm{kpc}$ as for the MS (Yoshizawa \& Noguchi 2003), the clumps have diameters of about $0.5 \ldots 0.8 \mathrm{pc}$ (QSO B1331+170) and 30 ..50 pc (QSO J0003-2323), respectively. From this, we are also able to estimate other distancedependent physical parameters such as mass and pressure of the gas. The total H I mass as a function of distance $d$ is given by

$M_{\mathrm{HI}}=m_{\mathrm{H}} d^{2} \tan ^{2} \phi \sum_{i} N_{\mathrm{HI}}^{(i)}$,

with $m_{\mathrm{HI}}$ being the mass of the hydrogen atom, $\phi$ the angular size of a resolution element (pixel) of the $N_{\mathrm{HI}}$ column density map (16" for QSO B1331+170 and 6" for QSO J0003-2323), and $N_{\mathrm{HI}}^{(i)}$ the detected column density for each pixel in the map. The mean density of each clump can be determined using the measured peak column density, assuming a spherical symmetry of the clumps and a constant particle density $n_{\mathrm{HI}}$ :

$n_{\mathrm{HI}}=\frac{N_{\mathrm{HI}}}{d \tan \phi}$.
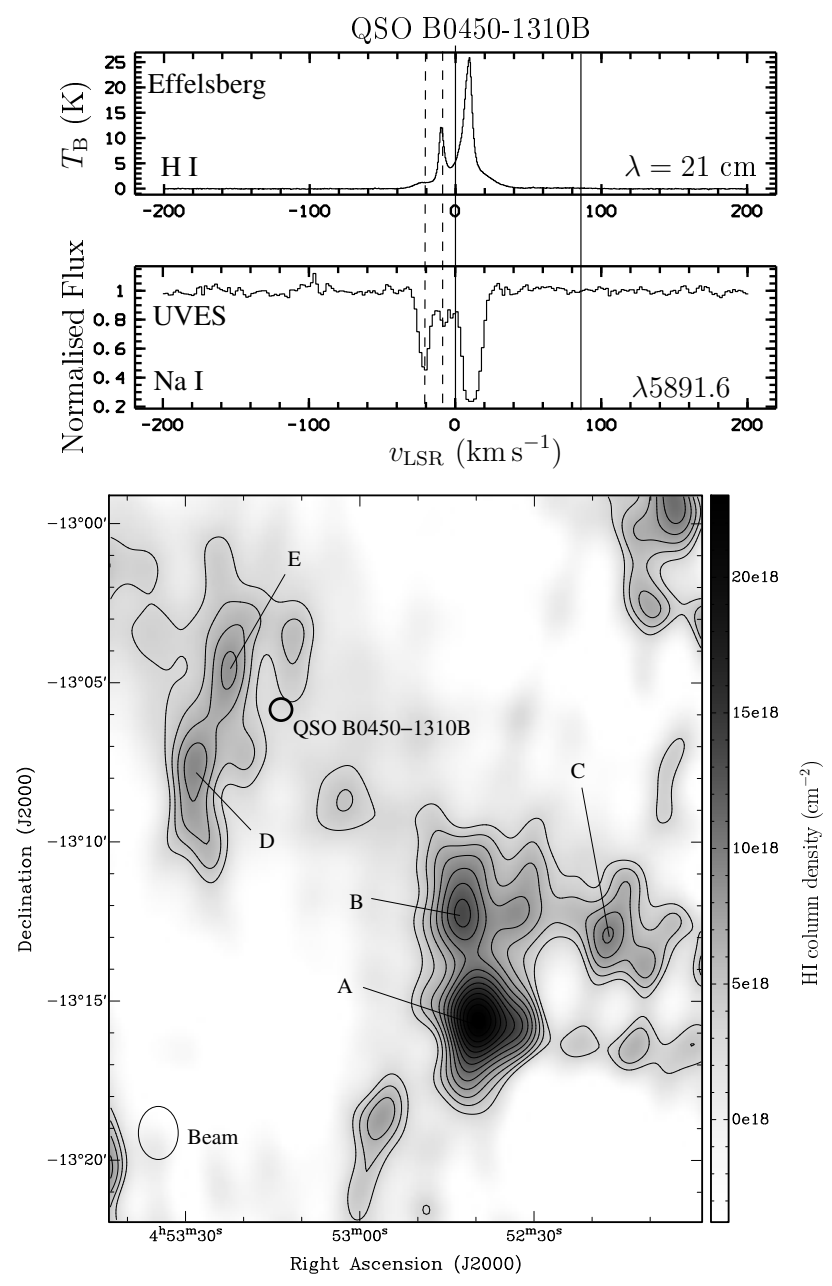

Fig. 6. Upper panel: Na I absorption spectra towards QSO B0450-1310B obtained with UVES. There is no Ca II spectrum available for this sight line. The corresponding Effelsberg H I emission line profile is shown. Lower panel: VLA 21-cm column density map of the low-velocity gas observed towards QSO B0450-1310B. The sightline passes the outer parts of clumps D and E. The contour lines are identical to those in Fig. 2 (right panel).

Using the upper limit for the kinetic temperature, the derived particle density, and assuming an ideal gas, one gets an expression for the upper pressure limit

$\frac{P}{k_{\mathrm{B}}}=n_{\mathrm{HI}} T_{\max }$

Inserting Eq. (2) into Eq. (3) provides the upper gas pressure limit (under the assumptions made before)

$\frac{P}{k_{\mathrm{B}}}=\frac{N_{\mathrm{HI}}}{d \tan \phi} T_{\max }=\frac{\sin b}{z} \frac{N_{\mathrm{HI}}}{\tan \phi} T_{\max }$

as a function of the (unknown) distance of the cloud, or as a function of height, $z$, above the plane (considering the Galactic latitude, $b$ ). However, one major uncertainty here is the determination of angular sizes of the clumps, which could have a great impact if the clumps are not resolved.

Table 4 shows the distance-dependent parameters for the clumps observed in the directions of QSO B1331+170 (1 kpc) and QSO J0003-2323 (50 kpc), for which a distance was assumed based on their association with known IVC or HVC complexes. The columns contain the spatial diameters, the masses, 

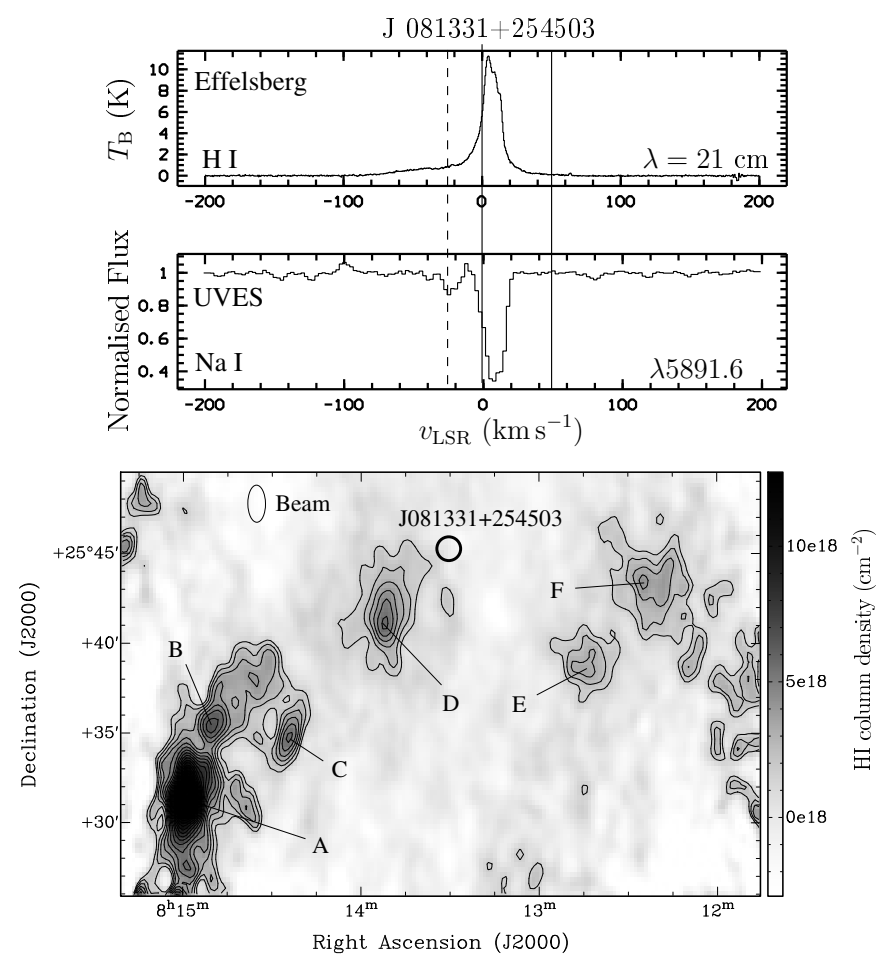

Fig. 7. Upper panel: Na I absorption and corresponding Effelsberg H I emission spectra in the direction of J081331+254503. No Ca II data are available for this sight line. Lower panel: WSRT 21-cm map of the intermediate-velocity gas in the direction of the quasar $\mathrm{J} 081331+254503$. The line of sight towards J081331+254503 passes the very outer envelope of clump D. The contour lines are identical to those in Fig. 1 (lower panel).

Table 4. Distance-dependent parameters of the small-scale structures observed towards QSO B1331+170 (WSRT) and QSO J0003-2323 (VLA).

\begin{tabular}{cccccc}
\hline \hline Quasar & clump & $r$ & $\begin{array}{c}M_{\mathrm{HI}} \\
{[\mathrm{pc}]}\end{array}$ & $\begin{array}{c}n_{\mathrm{HI}} \\
{\left[M_{\odot}\right]}\end{array}$ & $\begin{array}{c}\left(\frac{P}{k_{\mathrm{B}}}\right)_{\text {upper }} \\
{\left[\mathrm{cm}^{-3}\right]}\end{array}$ \\
{$\left[\mathrm{cm}^{-3} \mathrm{~K}\right]$}
\end{tabular}

the particle densities, and the upper pressure limits of the clumps.

The small line widths of the clumps, especially the ones towards QSO J0003-2323 (Sect. 3.1 and Table 3), indicate that the absorbing gas is cold with upper temperature limits of $T_{\text {kin }} \approx$ $100 \mathrm{~K}$ for the clumps with the most narrow line widths of about $\Delta v_{\mathrm{FWHM}} \approx 2 \mathrm{~km} \mathrm{~s}^{-1}$ and $T_{\text {kin }} \approx 3700 \mathrm{~K}$ for the structures with the most broad values of $\Delta v_{\mathrm{FWHM}} \approx 13 \mathrm{~km} \mathrm{~s}^{-1}$. The temperatures could be even lower because effects like turbulent motions within the gas likely contribute to the observed line width. The temperatures and projected sizes of the clumps detected in the direction of QSO B1331+170 and QSO J0003-2323 show that the clumps are compact and cold rather than diffuse and extended.

Because the smallest spatial structures observed are similar in size to the synthesised WSRT and VLA beams, it is possible that the observed clumps are actually not resolved and contain structures on even smaller scales. This raises the question whether IVCs and HVCs and their small-scale structures could have fractal properties, as discussed in Vogelaar \& Wakker (1994). However, the answer to this question requires many more observations at different spatial resolutions.

We can now compare the determined upper pressure limits for the high-velocity absorbing system in the direction of QSO J0003-2323 and QSO B1331+170 with the models of Wolfire et al. (1995). These authors calculated the thermal equilibrium gas temperature for HVCs in the Galactic halo as a function of height $z$ above the Galactic plane and find that stable two-phase gas exists only within a narrow pressure range. Both sources lie near the Galactic poles, so the distance roughly equals the Galactic height. Various values for metallicities and dust-to-gas ratios were used to take different IVC and HVC origins into account. Under the assumption that the observed HVC in the direction of QSO J0003-2323 is associated with the Magellanic Stream, we compared the derived upper pressure limit of $\left(P / k_{\mathrm{B}}\right)_{\text {upper }}$ with the pressure range of $P \approx 20 \ldots 180 \mathrm{~cm}^{-3} \mathrm{~K}$ for the HVC gas stripped from the MS, calculated using a metallicity of $Z \approx 0.4$ and a dust-to-gas ratio of $D / G=0.2$ (Wolfire et al. 1995). To remind the reader, $D / G$ refers to the mass of the small grain population, where $D / G=1$ implies that about $4 \%$ of the cosmic carbon is found in grains smaller than $15 \AA$.

The observed values of clump A and D are relatively low but consistent with the models. The other structures reveal pressures that are incompatible with the simulations. However, as discussed before, the physical size of the clumps might be overestimated (if unresolved). In that case, the derived upper pressure limit would be underestimated.

For QSO B1331+170, the simulated pressure range of $P \approx$ $150 \ldots 650 \mathrm{~cm}^{-3} \mathrm{~K}$ (assuming a Galactic fountain origin, a metallicity of about 1.0 solar, and a dust-to-gas ratio of $D / G=$ 0.3 ) or $P \approx 650 \ldots 3500 \mathrm{~cm}^{-3} \mathrm{~K}$ (metallicity of about 1.0 and a dust-to-gas ratio of $D / G=1.0$ ) is consistent with our observations.

For the two other sightlines toward QSO B0450-1310 and $\mathrm{J} 081331+254503$, the lack of distance information for the halo clouds in these directions unfortunately impedes a similar pressure analysis.

\subsection{On the metal abundances of the clumps}

To better understand the nature and the origin of these lowcolumn density, small-scale clumps in the halo and their relation to large IVC and HVC complexes, it would be very helpful to constrain the chemical composition of these objects. Our optical observations provide column density information for the two species $\mathrm{Ca}$ II and $\mathrm{Na}$ I, while the 21 -cm observations give $N(\mathrm{HI})$.

However, there are a number of systematic effects that inhibit any reliable estimate of the absolute calcium and sodium abundances in the clouds. In diffuse neutral gas, $\mathrm{Ca}$ II and $\mathrm{Na}$ I are not the dominant ionization stages of these elements because of their low ionisation potentials compared to hydrogen and both elements usually are depleted into dust grains. Therefore, the measured $\mathrm{Ca}$ II and Na I column densities in the halo absorbers are not expected to be representative of the total calcium and 
sodium abundances in the gas. Another severe systematic problem for determining the absolute abundances of these elements comes from the comparison of the absorption-line data with the H I 21-cm emission measurements. The beam sizes of the WSRT and the VLA still are very large compared to the pencil beam of the absorption measurements. Therefore, the H I column density values are just averages over a larger area on the sky, and the values at the quasar positions are not accurately known. In all our cases, the quasar sightlines are located outside or at the edge of the H I clumps. There, one might just observe the Gaussian decline of the point spread function in a region where there might not even be any detectable signal at all. Also, the radial velocities and the component structure of the absorption lines are different than those seen in 21-cm emission. This points towards smallscale structure in the gas that is not detected and/or resolved in the beam-smeared H I data.

Despite all these substantial uncertainties, ionic gas phase abundances, $A$, for $\mathrm{Ca}$ II and $\mathrm{Na}$ I (relative to $\mathrm{HI}$ ) have been published for a large number of Milky Way disk and halo absorbers (e.g., Wakker 2001; Wakker \& Mathis 2000), implying that, for each ion, $A$ scales with $\mathrm{HI}$ in a different manner and with different scatter. As an example, we compared the ionic gas phase abundance of $\mathrm{Na}$ in the halo absorber towards QSO B0450+1310B with these previous observations. We chose the QSO B0450+1310B sightlines as it passes relatively close to one of the H I clumps seen in the high- resolution 21-cm data (Fig. 3). For the halo absorber towards QSO B0450+1310B, a column density of $N_{\mathrm{HI}}=2.8 \times 10^{18} \mathrm{~cm}^{-2}$ was measured by the VLA $\left(N_{\mathrm{Na}}=10^{12} \mathrm{~cm}^{-2}\right)$. The associated Na I abundance is $A_{\mathrm{NaI}} \approx 360 \times 10^{-9}$, which is about seven times larger than $\mathrm{Na}$ I abundances previously measured in diffuse halo clouds $\left(A_{\mathrm{NaI}} \leq 50 \times 10^{-9}, A_{\mathrm{CaII}}=3 \ldots 350 \times 10^{-9}\right.$; Wakker 2001 ; Wakker \& Mathis 2000). However, Wakker (2001) points out that the Ca II (Na I) abundance in IVC and HVC gas varies by a factor of 2-5 (10) within and by a factor 5-10 (100) between clouds. Since all our clouds are generally of low column density, this substantial scatter makes it impossible to draw any meaningful conclusions about the $\mathrm{Ca}$ and $\mathrm{Na}$ abundances and overall metallicities of the absorbers based solely on our $\mathrm{Ca}$ II and $\mathrm{Na}$ I measurements.

A much more reliable method of studying the metal content of weak halo absorbers is to analyse UV and FUV spectra, as obtained with FUSE and HST/STIS. Richter et al. (2009) recently have studied the metallicities in seven low-column density, highvelocity absorbers by comparing the column densities of $\mathrm{O}$ I with those of $\mathrm{HI}$. The ratio $\mathrm{OI} / \mathrm{HI}$ is a very robust measure of the metallicity of the gas, as it does not depend on ionisation effects and dust depletion. In this study, $N_{\mathrm{H} \text { I }}$ has been derived from FUV absorption data by fitting the higher Lyman series lines in absorption against the quasars, but not from beam-smeared 21-cm emission observations. The UV measurements indicate that, similar to the large IVC and HVC complexes, low-column density halo clouds span an abundance range between 0.1 and 1.0 solar, suggesting multiple (galactic and extragalactic) origins for the absorbers. Also, relative abundance ratios (e.g., Fe/Si) have been used to constrain the enrichment history of these clouds (see Richter et al. 2009). Interestingly, the simultaneous detection of $\mathrm{Ca}$ II and other low ions such as O I, Si II, and Fe II in two high-velocity systems towards PHL 1811 imply that the high-velocity $\mathrm{Ca}$ II absorbers considered in this study, and the weak UV absorption systems studied by Richter et al. (2009) trace the same population of low-column density halo clouds.

\section{Conclusions}

In this paper we have presented high-resolution $\mathrm{H}$ I observations with the WSRT and the VLA for four lines of sight through the Galactic halo where we previously detected weak low-, intermediate-, and high-velocity gas in Ca II and Na I absorption. Along these four sightlines, we detected one high-velocity cloud (probably belonging to the Magellanic Stream) seen in $\mathrm{Ca}$ II and Na I absorption and 21-cm emission. The four other detected features show low-velocity gas $\left(v_{\mathrm{dev}}<30 \mathrm{~km} \mathrm{~s}^{-1}\right)$. One of these systems is likely associated with the IV spur, another one is eventually part of the LLIV arch, both being IVC complexes. For the two remaining clouds, we cannot in the end decide whether they are part of the Milky Way disk or are located in the (lower) Galactic halo. The major results of our study are:

1. Along all four sightlines our high-resolution H I observations reveal the presence of cold, compact (sub-pc scale, except those associated with the Magellanic Stream) neutral gas clumps associated with $\mathrm{Ca}$ II and $\mathrm{Na}$ I absorbers. The intermediate- and high-velocity systems are located in the Milky Way halo, whereas the low-velocity features are either located in the lower halo or in the Milky Way disk.

2. The detected clumps have low peak column densities of $N_{\mathrm{HI}} \leq 3 \times 10^{19} \mathrm{~cm}^{-2}$. This, together with their small angular sizes (1-3 arcmin, typically), suggest that these smallscale H I structures are unseen and/or unresolved in current H I 21-cm all-sky surveys.

3. The HI line widths of the clumpy structures are relatively narrow $\left(1.8 \leq \Delta v_{\mathrm{FWHM}} \leq 13 \mathrm{~km} \mathrm{~s}^{-1}\right)$, leading to upper limits for the kinetic temperature in the range $T_{\mathrm{kin}}^{\max }=70-3700 \mathrm{~K}$.

4. In all four directions, the quasar sightlines used for the absorption spectra pass the outer envelopes of the clumps or the inter-clump medium, but do not pass the clump cores. The narrow $\mathrm{Ca}$ II and $\mathrm{Na}$ I absorption along these sightlines demonstrates that additional small-scale structure exists at column densities below the detection limit of the high- resolution H I 21-cm observations.

5. The sky positions, as well as the radial velocities of the halo absorber towards QSO J0003-2323 and QSO B1331+170, indicate a possible association with the Magellanic Stream and IV Spur, respectively. By using available distance information for these halo clouds we are able constrain distancedependent physical parameters, such as gas mass and thermal pressure. The derived parameters match the values predicted by the models of Wolfire et al. (1995).

Our study indicates that a substantial degree of small-scale structure exists in the neutral galactic halo gas that can only be traced with high- resolution H I 21-cm observations and with quasar absorption-line spectroscopy. The combination of the various data sets shows how important measurements at different wavelengths and resolutions are for a more realistic view of the distribution and physical properties of neutral gas structures in the Milky Way halo.

Using radio synthesis telescopes, we will continue the search for small-scale structures that are not resolved with the Effelsberg telescope. Moreover, we will proceed with our effort to systematically study absorption features in the Galactic halo along quasar sightlines using optical instruments and future space-based UV spectrographs. Such combined high-resolution emission and absorption observations will be crucial for better understanding the spatial distribution of gaseous structure in the inner and outer haloes of the Milky Way and their relation to 
intervening quasar absorption-line systems at low and high redshift.

Acknowledgements. P.R. and N.B.B. acknowledge financial support by the German Deutsche Forschungsgemeinschaft, DFG, through Emmy-Noether grant Ri 1124/3-1.

\section{References}

Ben Bekhti, N., Brüns, C., Kerp, J., \& Westmeier, T. 2006, A\&A, 457, 917 Ben Bekhti, N., Richter, P., Westmeier, T., \& Murphy, M. T. 2008, A\&A, accepted, 806

Blitz, L., Spergel, D. N., Teuben, P. J., \& Hartmann, D. 1999, in Stromlo Workshop on High-Velocity Clouds, ed. B. K. Gibson, \& M. E. Putman, ASP Conf. Ser., 166, 125

Brüns, C. 1998, in Astronomische Gesellschaft Meeting Abstracts, 5

Brüns, C., Kerp, J., \& Pagels, A. 2001, A\&A, 370, L26

Bregman, J. N. 1980, ApJ, 236, 577

Ding, J., Charlton, J. C., \& Churchill, C. W. 2005, ApJ, 621, 615

Ford, H. A., McClure-Griffiths, N. M., Lockman, F. J., et al. 2008, ApJ, 688, 290 Fraternali, F., Binney, J., Oosterloo, T., \& Sancisi, R. 2007, New Astron. Rev., 51,95

Högbom, J. A. 1974, A\&AS, 15, 417

Heiles, C., \& Troland, T. H. 2003, ApJ, 586, 1067

Kalberla, P. M. W. 2003, ApJ, 588, 805

Kalberla, P. M. W., Dedes, L., Kerp, J., \& Haud, U. 2007, A\&A, 469, 511

Lockman, F. J. 2002, ApJ, 580, L47

Lockman, F. J., Murphy, E. M., Petty-Powell, S., \& Urick, V. J. 2002, ApJS, 140, 331
Majewski, S. 2004, in Satellites and Tidal Streams, ed. F. Prada, D. Martinez Delgado, \& T. J. Mahoney, ASP Conf. Ser., 327, 63

Mathewson, D. S., Cleary, M. N., \& Murray, J. D. 1974, ApJ, 190, 291

Muller, C. A., Oort, J. H., \& Raimond, E. 1963, C. R. Acad. Sc. Paris, 257, 1661

Oort, J. H. 1966, Bull. Astron. Inst. Netherlands, 18, 421

Quilis, V., \& Moore, B. 2001, ApJ, 555, L95

Richter, P. 2006, in Reviews in Modern Astronomy, ed. S. Roeser, 19, 31

Richter, P., Sembach, K. R., Wakker, B. P., \& Savage, B. D. 2001, ApJ, 562, L181

Richter, P., Westmeier, T., \& Brüns, C. 2005, A\&A, 442, L49

Richter, P., Charlton, J. C., Fangano, A. P. M., Ben Bekhti, N., \& Masiero, J. R. 2009, ApJ, 695, 1631

Savage, B. D., \& Massa, D. 1987, ApJ, 314, 380

Sembach, K. R., Savage, B. D., \& Massa, D. 1991, ApJ, 372, 81

Shapiro, P. R., \& Benjamin, R. A. 1991, PASP, 103, 923

Shapiro, P. R., \& Field, G. B. 1976, ApJ, 205, 762

Stil, J. M., Lockman, F. J., Taylor, A. R., et al. 2006, ApJ, 637, 366

Thom, C., Putman, M. E., Gibson, B. K., et al. 2006, ApJ, 638, L97 van Woerden, H., Schwarz, U. J., Peletier, R. F., Wakker, B. P., \& Kalberla,

P. M. W. 1999, Nature, 400, 138

Vogelaar, M. G. R., \& Wakker, B. P. 1994, A\&A, 291, 557

Wakker, B. P. 1991, A\&A, 250, 499

Wakker, B. P. 2001, ApJS, 136, 463

Wakker, B. P., \& Mathis, J. S. 2000, ApJ, 544, L107

Wolfire, M. G., McKee, C. F., Hollenbach, D., \& Tielens, A. G. G. M. 1995, ApJ, 453, 673

Wakker, B. P., Howk, J. C., Savage, B. D., et al. 1999, Nature, 402, 388

Wakker, B. P., York, D. G., Howk, J. C., et al. 2007, ApJ, 670, L113

Wakker, B. P., York, D. G., Wilhelm, R., et al. 2008, ApJ, 672, 298

Yoshizawa, A. M., \& Noguchi, M. 2003, MNRAS, 339, 1135 